\title{
PERSPECTIVES
}

VIEWPOINT

\section{Unravelling mononuclear phagocyte heterogeneity}

\section{Frédéric Geissmann, Siamon Gordon, David A. Hume, Allan M. Mowat and Gwendalyn J. Randolph}

Abstract | When Ralph Steinman and Zanvil Cohn first described dendritic cells (DCs) in 1973 it took many years to convince the immunology community that these cells were truly distinct from macrophages. Almost four decades later, the $D C$ is regarded as the key initiator of adaptive immune responses; however, distinguishing DCs from macrophages still leads to confusion and debate in the field. Here, Nature Reviews Immunology asks five experts to discuss the issue of heterogeneity in the mononuclear phagocyte system and to give their opinion on the importance of defining these cells for future research.

\section{0]} Myeloid cells are infamously heterogeneous. Do you think that this has led to confusion in the field when discriminating between macrophages and dendritic cells (DCs)?

Frédéric Geissmann. Most cells types in the body are identified by their origin, anatomical location, function, and phenotype. Myeloid cells are arguably no more heterogeneous than other cell types (such as lymphoid cells or epithelial cells), and therefore heterogeneity may not be solely responsible for the confusion between macrophages and DCs.

Confusion has arisen in part from the use of nonspecific cell surface markers, such as $\mathrm{CD} 11 \mathrm{c}$ (also known as aX integrin), as a surrogate for function, anatomical location, and lineage. This has led to the name 'DC' being used for several cell types, including bona fide classical DCs, as well as activated monocytes, tissue macrophages, interferon (IFN)-producing cells and even some natural killer (NK) cells and eosinophils.

Confusion has arisen in part from the use of nonspecific cell surface markers
Recent in vivo experiments in mice have increased our understanding of the development and functions of DC and macrophage subsets $^{1,12,15,64,65}$ and should reduce confusion. However, despite this progress in mice, corresponding human subsets are yet to be characterized. Numerous studies have attempted to recapitulate some of the heterogeneity of human DCs and macrophages in vitro. However, in vitro differentiation of blood monocytes or bone marrow cells does not recapitulate in vivo differentiation. For example, Langerhans cells and microglial cells may not renew from bone marrow ${ }^{2-4}$.

Siamon Gordon. As far as mononuclear myeloid cells are concerned, I recognize a family of closely related cells with a common origin, which branches into irreversibly differentiated sublineages, such as macrophages, DCs and osteoclasts ${ }^{5}$.

Part of the confusion in the field arises from the adaptability and plasticity of macrophages. As a result of their extensive receptor repertoire and versatile biosynthetic capacity, they are highly responsive to the microheterogeneity present in different tissue environments ${ }^{6}$. Furthermore, following constitutive or inflammation-induced migration between different compartments of the body, they undergo marked phenotypical modulation ${ }^{7}$. Even in the absence of inflammation and infection, macrophage populations ${ }^{8}$ display bewildering heterogeneity. For example, numerous mononuclear phagocyte populations are found in the mouse spleen, including red and white pulp macrophages, 'reservoirs' of mobilizable monocytes ${ }^{9}$, marginal zone metallophilic macrophages and outer zone macrophages, and further complexity arises from the presence of resident, motile and migratory activated DCs in the same organ ${ }^{10}$.

Another problem is the marked heterogeneity in the turnover and variable lifespan of macrophages - hours to weeks depending on stimulation, programmed cell death and/or injury. In the mouse, genetic manipulation and intravital imaging methods make it possible to study direct precursor-product relationships. However, the dynamics of tissue populations in humans remain largely unexplored.

Gwendalyn J. Randolph. Yes, there is confusion. However, I think the confusion can be resolved when the right considerations are given. First, confusion should be minimal when spleen and lymph nodes are considered. In these locations, where historically the pioneering work on DCs and to some extent macrophages has centred, it is clear that $\mathrm{CD} 11 \mathrm{c}^{\text {hi }}$ mononuclear phagocytes are DCs rather than macrophages. An abundance of recent evidence confirms that spleen and lymph node DCs are functionally distinct from macrophages, do not arise from monocytes, and share fewer properties with monocytes than macrophages do $\mathrm{d}^{11-17}$.

So here is where I see the contemporary confusion arising. DCs and macrophages in non-lymphoid organs are poorly characterized compared with their lymphoid tissue counterparts. At last, work in vivo on macrophage and DC biology in non-lymphoid organs is intense. Many of us have assumed that the same set of markers that definitively distinguish DCs from macrophages in lymphoid organs will also apply in nonlymphoid organs, but it is turning out that this premise is not true. So, I think the main confusion arises when scientists assume that what is true in lymphoid organs is also true in non-lymphoid organs and what is true under 


\section{The contributors}

Frédéric Geissmann gained a medical degree in 1996 and a Ph.D. in immunology in 1999, both from the University of Paris, France. After a postdoctoral fellowship with D. Littman in New York, USA, he started his own lab in Paris (the Unit 838 of Inserm) to study the in vivo development and functions of cells of the 'mononuclear phagocyte system' and their roles in the pathogenesis of inflammatory disorders. He moved his lab to London, UK, in 2008 and is currently the Arc Chair of inflammation Biology at King's College, London, and the head of the newly established Centre for Molecular and Cellular Biology of Inflammation.

Siamon Gordon is an Emeritus Professor of Cellular Pathology at the University of Oxford, UK. He obtained his medical degree at the University of Cape Town, South Africa, in 1961 and a Ph.D. at Rockefeller University, New York, USA, in 1971, under the tutorage of Z. A. Cohn. Since 1976, he has been at the Sir William Dunn School of Pathology at the University of Oxford, retiring from the Glaxo-Wellcome Professorship of Cellular Pathology in 2008. He spent a sabbatical at the US National Institute of Allergy and Infectious Diseases (NIAID) and the US National Cancer Institute (NCI).

David A. Hume (F.R.S.E.) is Director of The Roslin Institute and Research Director of the Royal (Dick) School of Veterinary Studies at the University of Edinburgh, UK. He is an international authority on macrophage differentiation and the function of macrophages in infection, inflammatory disease and cancer. He is especially interested in transcriptional regulation, transcriptional networks and the evolution of macrophage biology across vertebrate species. He did his Ph.D. at the Australian National University, Canberra, Australia, and his postdoctoral work with S. Gordon at the University of Oxford in the early 1980s. Before coming to Edinburgh, he was at the Institute for Molecular Bioscience at the University of Queensland, Australia, where he was Director of the Australian Research Council Special Research Centre for Functional and Applied Genomics.

Allan M. Mowat is Professor of Mucosal Immunology at the University of Glasgow, UK, where his laboratory is engaged in studies of macrophages and dendritic cells in the mouse intestinal mucosa. $\mathrm{He}$ is also involved in a large amount of undergraduate teaching and contributes to textbooks in general and mucosal immunology.

Gwendalyn J. Randolph completed her Ph.D. at the State University of New York in Stony Brook, New York, USA, in pathology and immunology. She then carried out postdoctoral research at the Rockefeller University and Weill Medical College of Cornell University, New York. She is now Professor in the Department of Gene and Cell Medicine and a member of the Immunology Institute at Mount Sinai School of Medicine, New York. Her laboratory focuses on the trafficking of monocytes, dendritic cells, and macrophages, including in the context of inflammatory diseases such as atherosclerosis.

homeostatic conditions is also relevant in inflammation. None of these lines should be blurred until we know more about these wonderful populations in different anatomical sites and tissue states.

David A. Hume. The mononuclear phagocyte system is a family of cells derived from a committed progenitor in the bone marrow $^{18}$. The number of subpopulations of mononuclear phagocytes one can define is an exponential function of the number of markers examine ${ }^{19}$; the heterogeneity is essentially infinite because each gene has its own intrinsic probability of being expressed ${ }^{20}$.

It is clear that the definition of DCs has changed with time. The original description of DCs described them as not actively endocytic and not part of the mononuclear phagocyte system ${ }^{18,19}$. CD11c, the favoured DC marker, was already known to be expressed by most tissue macrophages before the use of CD11c-reporter transgenes as markers of DCs, and of CD11 c-DTR (diphtheria toxin receptor) mice to 'selectively' ablate them ${ }^{19,21}$. Subsequently, cells that were morphologically and functionally active phagocytes were found to 'convert' into DCs ${ }^{22}$. This led to the concept of the immature DC and then of inflammatory or tumour necrosis factor and inducible nitric oxide synthase-producing DC (TIP DC) ${ }^{23}$. As noted also by G.J.R, there is no obvious distinction between these cells and resident or classically activated macrophages, respectively.

If we consider the DC, as originally defined by Ralph Steinman and Zanvil Cohn, it was defined functionally by its ability to stimulate in an allogeneic mixed leukocyte reaction (MLR $)^{24}$, and the distinction from a macrophage is clear. These DCs do not express the widely studied macrophage marker F4/80 (also known as EMR1), CD11b (also known as aM integrin) or Fc receptors, they are not phagocytic and they do not adhere to fibronectin; we used these criteria to isolate this form of DC from the intestinal lamina propria ${ }^{25}$. As for the rest, most mononuclear phagocytes in the gut wall, for example, are monocyte-derived, $\mathrm{F} 4 / 80^{+}$and express the macrophage colony-stimulating factor 1 receptor (CSF1R; also known as CD115) and all the known endocytic receptors $^{26-28}$; they have even been said to 'resemble macrophages ${ }^{26}$. But they have been termed DCs solely because they express CD11c. And that really does confuse the field.

Allan M. Mowat. In my own field of mucosal immunology, myeloid cell heterogeneity has led to considerable confusion, and many accepted truths probably need to be re-interpreted. In the gut, there are extraordinary numbers of cells of myeloid origin present in the absence of inflammation. There is a consensus that both macrophages and DCs in the gut are generally hyporesponsive $\mathrm{e}^{29,30}$, and this overall conclusion holds irrespective of what one calls the cells, but recent years have introduced increasing complexity, particularly in terms of DC biology $\mathrm{y}^{31}$.

More and more individual DC subsets have been defined based, for example, on their ability to induce the differentiation of $\mathrm{T}$ helper $17\left(\mathrm{~T}_{\mathrm{H}} 17\right)$ cells or forkhead box $\mathrm{p} 3$ $(\mathrm{FOXP} 3)^{+}$regulatory $\mathrm{T}\left(\mathrm{T}_{\mathrm{Reg}}\right)$ cells, promote IgA class-switching in B cells or produce pro-inflammatory factors, such as tumour necrosis factor (TNF), interleukin-23 (IL-23) and nitric oxide (NO) $)^{27,29,32-34}$. Almost without exception, it is claimed that specific DC or macrophage populations may be responsible for these diverse properties, and it is implied that these could be manipulated specifically for practical purposes, such as vaccine development and disease therapy. Many of these so-called DC subsets are not clearly defined and it is essential to understand that different groups use different methods to obtain and characterize DCs. Often the starting populations are pre-selected on the basis of arbitrarily defined levels of expression of markers that have been assumed to be specific for either DCs or macrophages, but are actually expressed by both ${ }^{35}$.

\section{it is essential to understand that different groups use different methods to obtain and characterize DCs}

Similar reservations cast doubt on the widely accepted view that a specific population of DCs can take up antigens from the intestinal lumen by sending processes through the epithelium ${ }^{36,37}$. Supported by 
elegant imaging data, these findings provoked great interest throughout the field and in the wider immunological community. Textbooks describe the phenomenon, and many experimental studies on vaccines, inflammation or infection rely on it as a starting paradigm. However, these DCs have been characterized entirely by their expression of $\mathrm{CX}_{3} \mathrm{C}$-chemokine receptor 1 $\left(\mathrm{CX}_{3} \mathrm{CR} 1\right)$ and MHC class II, rather than on any functional criteria. In fact, these cells seemingly cannot migrate to draining lymph nodes, or present antigen to specific T cells ${ }^{35}$.

I think that these 'sampling' cells may be tissue-resident macrophages, contributing little or nothing to shaping primary $\mathrm{T}$ cell responses and being responsible more for scavenging functions in the mucosa itself. These are distinct properties, and it is now essential that all previous work in the field is re-examined taking into account the most recent advances in phenotyping and functional characterization. Similar overlaps are emerging from studies of myeloid subsets previously thought to be distinct populations in lung, skin and other organs.

\section{T Are there any good phenotypical markers for DCs and macrophages, or should the distinction be based solely on function? If so, what does a DC do that a macrophage can't and vice versa?}

G.J.R. Generally, cells with high surface levels of F4/80 are macrophages, but lower levels of F4/80 are commonly observed on DCs. Furthermore, much has been made of CD11c as a marker for mouse DCs but, as mentioned above, the accuracy of this marker as a means to track DCs depends on the anatomical site in question. Myeloid cells with high levels of CD11c, though not those with low to intermediate CD11c, are DCs in lymphoid organs. However, in the lung, high levels of CD11c are found on macrophages ${ }^{38,39}$, and there are plenty of other anatomical locations outside of lymphoid organs where macrophages are CD $11 c^{+}$. Moreover, CD11b was once thought to be a universal, albeit not specific, marker for macrophages. However, it turns out that splenic macrophages are CD11b low or negative $e^{40}$, as are pulmonary macrophages ${ }^{38,39}$. There are a handful of C-type lectins that seem to be specifically expressed by DCs, but there is currently not enough knowledge to make definitive claims about any marker combination, especially in non-lymphoid compartments.
Perhaps the best functional attribute I can think of to distinguish DCs and macrophages is the property of DCs to localize in the $\mathrm{T}$ cell zone of lymphoid organs where they can stimulate $\mathrm{T}$ cells. This attribute combines several features that DCs seem to specialize in - potent, though not exclusive, ability to activate $\mathrm{T}$ cells and the ability to migrate into the $\mathrm{T}$ cell zone itself. Indeed, if I can paraphrase a comment I heard from Marc Jenkins (personal communication): one of the landmark contributions brought about by Ralph Steinman's discovery of DCs was how it highlighted the specialized nature of the $\mathrm{T}$ cell zone. As for migration, my favourite research subject, I am unceasingly impressed by experiments that compare DCs and macrophages in their ability to migrate - DCs are far superior. Indeed, evidence that $\mathrm{CX}_{3} \mathrm{CR} 1{ }^{\text {hi }} \mathrm{CD} 11 \mathrm{~b}{ }^{\text {hi }} \mathrm{MHC}$ class $\mathrm{II}^{+} \mathrm{CD} 11 \mathrm{c}^{+}$cells that sample the intestinal lumen (so-called lamina propria DCs) (6,41 $^{36}$ do not obviously migrate to mesenteric lymph nodes efficiently ${ }^{26,39}$ is one reason why these cells may be better classified as macrophages instead of their present classification as DCs.
In the long term, functional properties such as antigen processing and presentation or migration are too fluid to serve as strict barriers for giving a cell its name. Ongoing and future developments will make it possible to define DCs and macrophages by lineage. Classification according to lineage would somewhat disrupt the current functional and marker-based classification. For example, we know that monocyte-derived cells can develop properties of DCs, including potent antigen presentation and migratory abilities, and we currently call some monocyte-derived cells DCs. Nonetheless, I think that the best solution to integrating DC and macrophage biology in the future is to define DCs as those cells that arise from dedicated DC precursors that we know exist ${ }^{17}$, but which need more characterization and study, and to define macrophages as those cells that descend from either embryonic macrophages or blood monocytes. Communicating science is obviously easier when cells have a fitting name, but we shouldn't be afraid to let the names evolve to fit contemporary advances in our knowledge and to ultimately make further classification easier as the field advances.

\section{Glossary \\ CD11c-DTR (diphtheria toxin receptor) mice Mice genetically engineered to express the diphtheria toxin receptor under the control of the CD 11 c promoter. Following administration of diphtheria toxin, cells expressing $\mathrm{CD} 11 \mathrm{c}$ are transiently depleted in these \\ Microglial cell \\ A phagocytic cell of myeloid origin that is involved in the innate immune response in the central nervous system. Microglial cells are thought to be the brain-resident macrophages.} animals. These mice have typically been used to study DC function, but certain $\mathrm{CD} 11 \mathrm{C}^{+}$macrophage populations are also depleted by diphtheria toxin treatment.

\section{Cross-presentation}

The mechanism by which certain APCs take up, process and present extracellular antigens on $\mathrm{MHC}$ class I molecules to stimulate CD8 ${ }^{+} \mathrm{T}$ cells. This property is atypical, as most cells exclusively present peptides derived from endogenous proteins on MHC class I molecules.

\section{Kupffer cell}

A specialized macrophage that lines the sinusoidal vessels of the liver. These cells regulate local immune responses, and remove microbial particles, endotoxin and other noxious substances that penetrate the portal venous system.

Langerhans cell

A type of DC that is resident in the epidermal layer of the skin.

M1 macrophages

A macrophage subtype that produces pro-inflammatory cytokines and has cytotoxic functions.

\section{M2 macrophages}

A macrophage subtype that acts to dampen inflammatory responses and scavenge debris, as well as to promote angiogenesis and tissue remodelling and repair.

Marginal zone metallophilic macrophage A type of macrophage that surrounds the splenic white pulp, adjacent to the marginal sinus, and is involved in trapping particulate antigens.

\section{Mixed leukocyte reaction}

(MLR). A tissue-culture technique for testing T cell cultured with MHC-mismatched APCs, and proliferation of the $T$ cells is determined by measuring the incorporation of ${ }^{3} \mathrm{H}$-thymidine into the DNA of dividing cells.

Myeloid-derived suppressor cells

(MDSCs). A group of immature CD 11 b+GR1+ cells (which include precursors of macrophages, granulocytes, DCs and myeloid cells) that are produced in response to various tumour-derived cytokines. These cells have been shown to induce tumour-associated antigen-specific CD8+ T cell tolerance.

\section{Osteoclast}

Multinucleated giant cells, of myeloid origin, that are responsible for bone resorption. Osteoclasts degrade bone matrix and solubilize calcium from bone.

\section{Plasmacytoid DCs}

An immature DC with a morphology that resembles that of a plasma cell. Plasmacytoid DCs produce type I IFNs in response to viral infection.

Tumour necrosis factor and inducible nitric oxide synthase-producing DC

(TIP DC). Monocyte-derived DCs that produce high quantities of tumour necrosis factor and nitric oxide.

These cells develop in mice from GR $1^{+}$monocytes during infection with certain bacteria, such as Listeria monocytogenes, or following myocardial damage. reactivity and APC activity. A population of T cells is 
A.M.M. The big problem we all encounter is that the conventional markers used to identify macrophages and DCs in mice, such as F4/80, CD11c, CD11b and MHC class II, have turned out not to be specific. This seems to be even more of an issue in tissues such as the respiratory tract and intestine, where the immunological environment is different to lymphoid tissue and there is continual antigen exposure.

Recent work shows that $\mathrm{CD} 103$ (also known as $\alpha \mathrm{E}$ integrin) and $\mathrm{CX}_{3} \mathrm{CR} 1$ expression define distinct populations of myeloid cells in the intestinal mucosa, although the exact nature of some of the subsets identified is contentious ${ }^{26,35,42}$. There is a population of $\mathrm{CD} 11 \mathrm{c}^{+} \mathrm{MHC}$ class $\mathrm{II}^{+}$cells expressing CD103, which have become the prototypic 'mucosal' DCs, inducing gut homing molecules and FOXP3 expression in naive $\mathrm{T}$ cells by producing retinoic acid $^{29}$. The cells expressing CX 3 CR1 are less well defined. On the basis of the expression of MHC class II and CD11c, some groups have separated this population into conventional macrophages and a subset of DCs with functions and origins distinct from the $\mathrm{CD}_{103^{+}} \mathrm{DCs}^{26,42,43}$. However closer examination shows that many, if not all, $\mathrm{CX}_{3} \mathrm{CR} 1^{+}$cells in gut mucosa express MHC class II and some CD11c, making these rather inappropriate distinguishing markers. In most respects, the $\mathrm{CX}_{3} \mathrm{CR}^{+}$ population actually seems to be generally homogeneous and is indistinguishable from tissue macrophages ${ }^{35}$.

Most importantly, recent work shows that $\mathrm{CX}_{3} \mathrm{CR}^{+}$cells in the mucosa do not migrate from the mucosal tissue to the draining mesenteric lymph nodes or present antigen to naive T cells - only the CD103+ subset of DC can do this ${ }^{35}$. I think DCs and macrophages should be distinguished functionally in this way, as I am not persuaded that macrophages can migrate and activate naive $\mathrm{T}$ cells to any great extent in normal conditions. Rather, their functions are more related to proteolytic and catabolic activities, such as destruction of pathogens, scavenging of cellular debris and tissue remodelling.

F.G. Phenotypic markers or functions alone are usually not sufficient. As for any other cells, macrophages and DCs should be characterized by a combination of cellular origin, anatomical location, function and phenotype. For example, alveolar macrophages express CD11c, but are best defined by their anatomical location and their morphology. Anatomical location is also crucial to identify microglial and Kupffer cells. Classical DCs are best characterized by their location in defined areas of lymphoid organs and are uniquely specialized in processing exogenous antigen for presentation to $\mathrm{T}$ cells and $\mathrm{T}$ cell priming. Plasmacytoid DCs are poor antigen presenting cells, but produce and secrete large amounts of type 1 IFN following Toll-like receptor 9 (TLR9)-signalling. The question implies a dichotomy of DCs and macrophages that may not be relevant to biology. Both DCs and macrophages consist of distinct subsets, and have many functions. The paradigmatic DCs of textbooks live in the periphery, uptake antigen, migrate from the periphery to the lymph node and initiate the immune response. This cell resembles the Langerhans cell, and the ability to migrate and carry antigen has been proposed as a criterion to define DCs. Macrophages are best defined by their phagocytic activity and are generally thought to be tissue-resident cells.

However, recent evidence indicates that macrophages can migrate, and that Langerhans cells are not important for $\mathrm{T}$ cell priming ${ }^{44}$. Also, although some macrophages (for example, Kupffer cells) are actively phagocytic, other macrophages (for example, microglial cells) show only weak phagocytic ability. So we do not really have any good functional criteria for defining macrophages and DCs as they represent not just two distinct populations, but various cell types.

S.G. There is no single morphologic or protein marker of macrophages or DCs which is unambiguous. CD11c expression, for example, is also abundant on alveolar macrophages, yet these cells have poor expression of F4/80, a widely used mouse macrophage antigen marker. Additionally, the F4/80 antigen is readily detected on immature DCs, although it is downregulated on DCs after maturation. CD11b can be present or absent on tissue macrophages in situ.

\section{Not enough attention has} been paid to the expression of antimicrobial effector molecules

Commonly, high constitutive expression of MHC class II is used to define mature DCs in the mouse. Resident macrophages in the mouse do not express MHC class II antigens until they are activated by IFN $\gamma$. However, MHC class II is constitutively present on human tissue macrophages, even in the absence of overt infection.
Although flow cytometry has been of great help in defining lymphocyte heterogeneity, embedded tissue macrophages are fragile and difficult to isolate from solid organs, even after enzymatic tissue digestion. In situ immunochemistry is an art and a qualitative tool, sensitive to fixation conditions, even with amplification and retrieval methods, especially when monoclonal antibodies are used. Functional analysis is a sine qua non for defining DCs, but it does not lend itself to single-cell studies.

Not enough attention has been paid to the expression of antimicrobial effector molecules, such as lysozyme ${ }^{45}$, which is abundant in neutrophils and also readily secreted by monocytes and macrophages, but is only poorly expressed, if at all, by DCs. It is hoped that microarray and proteomic analysis will yield new markers to detect proteins that distinguish macrophages from DCs, both in mice and in humans. However, no single set of markers can be expected to apply to all stages of cell differentiation and activation.

In characterizing macrophages and DCs, I like to use the analogy of a surviving text fragment attributed to Archilocus, a Greek philosopher, and made famous by Isaiah Berlin ${ }^{46}$ among others: "The fox knows many things, the hedgehog knows one big thing". To my mind, the macrophage is the fox, able to carry out a several functions, whereas the DC is specialized for one big function, namely to activate naive T lymphocytes ${ }^{10}$.

D.A.H. The DC doctrine is that DCs can uniquely present antigen to naive T cells, so a cell that can do this is, by definition, a DC. I feel this viewpoint is untenable. Myeloid antigen-presenting cells (APCs) have various APC activities that can stimulate a range of effector T cell outputs. The $\mathrm{F} 4 / 80^{+} \mathrm{CD} 11 \mathrm{~b}^{+}$ macrophages that we isolate from the intestinal lamina propria do not activate T cells in MLRs; they actually suppress the response. But it has been argued that intestinal $\mathrm{F} 4 / 80^{+} \mathrm{CD} 11 \mathrm{~b}^{+}$myeloid APCs can stimulate the development of FOXP $3^{+} \mathrm{T}_{\mathrm{Reg}}$ cells ${ }^{27}$. Another study showed that the cells we call macrophages (the authors called them

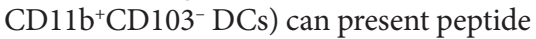
and activate naive OT-II transgenic $\mathrm{CD} 4^{+}$ $\mathrm{T}$ cells ${ }^{26}$. It is not clear that this assay has the same APC requirements as the MLR.

The problem really arises with in vitro assays ${ }^{21}$. In my opinion, macrophages as some define them are actually mixtures of stimulatory and inhibitory cells from different areas of the primary organs, whereas DCs are a minor population of cells exclusively from the stimulatory end of the 
myeloid APC continuum ${ }^{21}$. The unique function is actually at the 'macrophage' end of this continuum; I think that these are the cells that generate $\mathrm{T}$ cell tolerance. The DC believers term macrophages that can suppress T cell responses 'tolerogenic' $\mathrm{DCs}^{47}$ !

So, the problem is one of nomenclature. The term DC should be used exclusively for the APCs that reside in $\mathrm{T}$ cell areas of spleen and lymph node and the small numbers of similar cells at mucosal surfaces. All the contributors agree that these cells are not phagocytic, and probably not descended from cells that ever were phagocytic. We simply need to accept that some macrophages, defined by origin, CSF1-dependence in vivo, markers - such as F4/80, CD11b and Fc receptors and phagocytic activity, can in fact present antigen to naive $\mathrm{T}$ cells and the controversy evaporates.

\section{What culture conditions should a} researcher use to generate macrophages and DCs? How do the in vitro-generated and in vivo cells compare?

A.M.M. Like others, we use the simple and conventional methods of growing bone marrow cells in either granulocyte-macrophage colony-stimulating factor (GM-CSF) or CSF1 (also known as M-CSF) to obtain mouse DCs and macrophages, respectively. These methods have the advantage of generating reasonably homogeneous and reliable numbers of cells which can be manipulated in vitro.

However, the cells obtained are probably not representative of their counterparts in tissues. Their early stage of differentiation and homogeneity are unlikely to be replicated in vivo. More importantly, they have not been exposed to any of the environmental factors known to regulate myeloid cell differentiation and function in tissues. For example, DCs and macrophages in the intestine show many unusual properties compared with their equivalents in other tissues, blood or bone marrow and these are known to be conditioned by local factors ${ }^{29,30,48}$. However, the mediators involved are still being identified and no in vitro conditions have yet been found that replicate the full characteristics of the populations in situ. The same issues apply to other tissues, and so real advances will only be made using cells isolated from the organs themselves.

F.G. DCs obtained in culture from monocytes or bone marrow precursor cells exist in two functionally and phenotypically distinct states, immature and mature. Fundamental progress in understanding the cell biology of antigen presentation and the cellular mechanisms that allow DCs to initiate immunity or promote tolerance has been made using these cells ${ }^{49}$.

Macrophages can also be obtained from monocytes or bone marrow precursors following culture with CSF1. This experimental model has allowed investigators to identify cytokines and bacterial products that control their effector functions. M1 macrophages, the prototypical activating stimuli of which are IFN $\gamma$ and lipopolysaccharide (LPS), exhibit potent microbicidal properties, whereas M2 macrophages support $\mathrm{T}_{\mathrm{H}}$ 2-type effector functions and may play a role in resolving inflammation through endocytic clearance and trophic factor synthesis ${ }^{50}$.

However, the heterogeneity of the mononuclear phagocyte system is poorly recapitulated by these in vitro models. In other words, in vitro-generated macrophages and DCs, albeit useful to study the cell biology of phagocytosis and antigen presentation, for example, do not represent a model to study the specialized functions of the diverse cell types that are present in vivo, or the regulation of their development and functions by the tissue microenvironement. As an example, many tissue macrophages and DC subsets do not derive from blood monocytes, and some may not even derive from the bone marrow ${ }^{1,4}$. In vivo studies are required to analyse the functions of the mononuclear phagocyte system.

D.A.H. Individual colonies produced in vitro from bone marrow in GM-CSF contained classical DCs as well as granulocytes and macrophages $^{51}$. Bone marrow contains a common progenitor (the macrophage and DC progenitor $\left.(\mathrm{MDP})^{52}\right)$, and a so-called common DC progenitor (CDP) ${ }^{15}$. CDPs are probably identical to high-proliferative potential colony-forming cells (HPP-CFC) that require more than one factor to produce colonies ${ }^{19}$. There are at least seven cytokines that one should consider in studying myeloid APC differentiation; CSF1, GM-CSF, granulocyte colony-stimulating factor (G-CSF), IL-3, FMS-related tyrosine kinase 3 ligand (FLT3L), IL-4 and IFN $\gamma$.

A dogma has emerged that cells grown in GM-CSF are DCs, and those grown in CSF1 are macrophages ${ }^{19}$, but cells grown in both of these conditions are phagocytes and have been studied as functionally distinct macrophages $^{53}$. Both cell types are in vitro artefacts; in vivo, progenitors do not encounter any of these factors in isolation, and there are no obvious in vivo counterparts to the in vitroderived cells. HPP-CFCs require a combination of IL-3, GM-CSF, CSF1 and IFN $\gamma$ for optimal proliferation to differentiate into macrophages $^{19}$. Although DCs were originally thought to be CSF1-independent, this is clearly not the case ${ }^{54}$ and antibody against CSF1R is now used for the purification of DC progenitors ${ }^{15}$.

FLT3, the receptor for FLT3L, is a marker for haematopoietic stem cells and common myeloid progenitors, and it is retained on classical DCs in the spleen. FLT3L can expand myeloid APC populations in vitro and in vivo ${ }^{15}$ but, like CSF1, probably acts on the HPP-CFC in combination with other factors.

G.J.R. DCs and macrophages derived in culture show functional properties generally consistent with their counterparts in vivo. However, they are only approximations of cells that exist in vivo. They are useful for cell biological studies, for migration studies to some extent and in some cases they hold valuable promise as agents of cell-based immune therapies. Cultured DCs fuelled the growth of the DC field in the mid-1990s, so they are historically important.

Now, the DC field has moved beyond this phase and makes use of cultured DCs in combination with in vivo models. Generally, DCs cultured in GM-CSF are thought to be counterparts of inflammation-derived DCs, but this needs to be formally shown.

I have less experience with cultured macrophages. Perhaps they are over-used in studies of M1 and M2 polarization states, and peritoneal macrophages isolated by peritoneal lavage may too often be thought to model macrophages from any anatomic site.

However, overall, I think the field appropriately uses cultured DCs and macrophages without over-extending the interpretation of the data generated from them.

S.G. The ability to generate large numbers of DCs from mouse bone marrow and from human blood monocytes with the aid of growth-factor cocktails has proved irresistible to investigators for obvious reasons; for example, the direct isolation of these cells from blood and tissues is tedious and often results in poor yields and the introduction of artefacts.

Less well appreciated is the rapid 'acculturation' of macrophages that occurs ex vivo. Kupffer cells, for example, do not express CD11b in situ, but rapidly acquire this complement receptor in cell culture. Even modifying culture vessels with media and growth factors cannot prevent this artefact of isolation. Alveolar macrophages are round and loosely adherent in vivo, but are profoundly altered in morphology by adherence to 
tissue culture plastic, as are all macrophages. The use of undefined, non-physiological supplements such as fetal bovine serum is particularly egregious, and defined media should be used wherever possible.

In situ analysis is therefore mandatory, but DCs and macrophages in the tissue can be difficult to access, quantify or test functionally. Therefore an integrated approach, with appropriate awareness of artefact, is required. My own fantasy is to be able to recreate the full panoply of macrophage phenotypes observed in vivo, from embryonic stem cells and other haematopoietic stem cells, entirely in vitro. This will require knowing their in situ phenotype in detail and elucidation of the cellular and extracellular environment in different organs, such as the liver, gut, lung, brain and uterus.

\section{How important is the issue of defining macrophages and DCs for future} research and potential immune therapies?

D.A.H. It is my view, discussed in detail elsewhere ${ }^{18-21}$, that all cells of the mononuclear phagocyte system can interact with $\mathrm{T}$ cells. It does not matter whether they are called macrophages or DCs, what matters is the outcome of their interaction in a pathological or therapeutic context. Antigen presentation is a regulated function. We can manipulate APC activity empirically using adjuvants such as microbial products or various cytokines. There are no markers that predict in vivo APC function other than high expression of MHC class II and co-stimulatory molecules and cytokine expression. By purifying out the suppressive 'macrophages', we may succeed in getting a population of DCs that is more effective in a particular $\mathrm{T}$ cell activation assay in vitro, but classical DCs are a small subset of the potential APCs. Most of the active APCs for adoptive immunotherapy are in the macrophage fraction ${ }^{21}$.

I think that it is not possible to define macrophages and DCs as separate entities; they are a continuum of progeny of a common precursor. The suggestion that DCs are a separate lineage is clearly wrong ${ }^{20}$. The DCs defined by Steinman and Cohn are cells of the mononuclear phagocyte system that occupy a particular niche in lymphoid tissues, no more or less unique than the specialized cells of the mononuclear phagocyte system found in other organs (for example, Kupffer cells and microglia). At mucosal surfaces, mononuclear phagocytes are adapted to sample, process and present antigen. There is an almost complete overlap between expression of MHC class II and the macrophage marker F4/80 in mononuclear phagocytes found in mucosal sites ${ }^{55}$. More progress would be made by focusing on the functions on genes and gene products; the infinite subdivision of myeloid cells using multicolour flow cytometry with arbitrary gates is a futile and unproductive avenue for future research.

\section{it is not possible to define macrophages and DCs as separate entities; they are a continuum of progeny of a common precursor}

F.G. Because macrophages and DCs consist of several cell types with discrete and essential functions, defining the development and functions of these cells in their natural in vivo context (particularly in humans) is essential for our understanding of innate and adaptive immune response, and is therefore necessary to improve treatments for inflammatory and infectious diseases, as well as for cancer. The success of modern vaccines and immune therapies has already benefited from an improved knowledge of macrophage and DC biology $^{56}$. Increasing our understanding of the functions of human macrophages and DCs in vivo is likely to be crucial for the design of new therapeutic approaches, but this task is challenging.

One of the key challenges is to translate knowledge from mouse studies to the human system. For example, we, and others, have proposed that mouse $\mathrm{GR} 1^{+}$monocytederived TIP DCs ${ }^{23}$ that arise following infection with Listeria monocytogenes or during myocardial damage, resemble human M1 macrophages, whereas the mouse cells derived from $\mathrm{GR}^{-}$monocytes resemble human M2 macrophages ${ }^{1,52,57}$. However, although TIP DCs and M1 macrophages share several functions and express common genes, one must keep in mind that M1 macrophages are human cells obtained from culture in vitro, but TIP DCs are mouse cells that differentiate in vivo. Of note, splenic $\mathrm{GR}^{+}$monocytes can differentiate into myeloid-derived suppressor cells (MDSCs) that promote the development of tumourinduced regulatory $\mathrm{T}$ cells and $\mathrm{T}$ cell anergy in tumour-bearing mice ${ }^{58}$.

Therefore, it may be naive to postulate that human M1 macrophages will always behave like TIP DCs in vivo. Indeed, it is likely that many of the in vivo functions of human macrophages and DCs will be difficult to predict from the in vitro study of human cells or from mouse studies alone.

A.M.M. I'm old fashioned and reductionist enough to think that selective therapeutic immunomodulation will only be possible if the relevant cells and molecules are identified accurately. In the case of macrophages and DCs, there is ample evidence that distinct functions may correlate with different phenotypic markers such as CD11b, F4/80, $\mathrm{CX}_{3} \mathrm{CR} 1, \mathrm{CD} 103$ and $\mathrm{CD} 8 \alpha$. Without doubt, this needs to be taken into account if their properties are to be exploited in practice. For instance, if DCs are the only cells that can prime and shape naive $\mathrm{T}$ cell functions, targeting them will be essential; for example, for developing novel vaccines, enhancing antitumour responses and inhibiting autoimmune responses.

A number of therapies are already being developed that target DC surface molecules, and it could be equally useful to apply similar strategies to the phenotypic subsets of DC which seem to have distinct functions or which operate in different tissues. The $\mathrm{CD}_{103^{+}}$population of intestinal DCs is an excellent example of this, as it has a crucial role in shaping mucosal immune responses by the generation of gut homing $\mathrm{T}_{\text {Reg }}$ cells $^{29}$. In the skin, an apparently similar subset can potently cross-present antigens to $\mathrm{CD}^{+}$ $\mathrm{T}_{\mathrm{C}}$ cells $\mathrm{s}^{59}$. These properties are being explored as targets for vaccination or induction of tolerance.

Conversely, macrophages may be more important to target for their pro-inflammatory and catabolic functions, without necessarily interfering with the initiation phase of immune responses. For all these reasons, establishing cell-type specific phenotypic or functional markers will be essential.

S.G. Macrophages and DCs provide attractive targets for therapy. Selective targeting of recruited or resident DCs or macrophages, or of effector cytokines, should not incapacitate the entire innate defence system against pathogens or disrupt the homeostatic functions of macrophages. The ability to harness DC antigen-presentation through enhanced vaccination strategies could transform tumour immunization and overcome lymphoid and myeloid suppressor mechanisms.

The increased understanding of patternrecognition receptor signalling will aid the development of better adjuvants to boost adaptive immune responses. Targeting immunogens to particular DC subsets (for example, through the use of monoclonal 
antibodies specific for molecules such as DEC205 (also known as Ly75) ${ }^{60}$ ) has shown promise in mouse models, although much more work is required to confirm these studies in primates and humans. Long-acting inducers of DC type-I IFN production, such as the TLR3 ligand polyinosinic-polycytidylic-poly-L-lysine (poly I:C:LC), need further evaluation ${ }^{61}$.

To be practical and effective, I think that it is necessary to target specific subsets of DCs or macrophages in vivo, rather than by ex vivo manipulation and subsequent adoptive transfer of cells. The ability to do so safely will depend on an improved knowledge of the molecular basis of mononuclear myeloid cell heterogeneity in vivo.

G.J.R. Both macrophages and DCs have crucial roles in events that influence immunity, health of tissues, and recovery from disease states. I don't see how research on any cell type can occur optimally without further defining the cell of interest, and all cells have to be called something.

\section{DC and macrophage biologists don't talk with each other enough. Indeed, if a cell is called a DC in a paper, I think that it is less likely that a macrophage biologist will read it and vice versa}

Right now, a limitation to progress in immunology - and by extension, immunological therapies - is that DC and macrophage biologists don't talk with each other enough. Indeed, if a cell is called a DC in a paper, I think that it is less likely that a macrophage biologist will read it and vice versa. If a neutral term is used in a paper, such as 'CD $11 c^{+}$ cell' ${ }^{62}$ or 'antigen-presenting cell', this may also limit or alter its impact by limiting the ability of the right scientists to find it.

Matt Collin, whose work focuses on human DCs and macrophages ${ }^{63}$, has suggested to me that M1 or classically activated macrophages are probably the same cells as TIP DCs (M. Collin, personal communication). Indeed, I cannot find any instance where the same study shows both cell types in the same place. This is just one example of how further work and open dialogue in defining macrophages and DCs could lead to a clearer and more comprehensive body of literature for all scientists. That, in turn, can only help to advance the field.
Frédéric Geissmann is at King's College London, Guy's Campus, London SE1 1UL, UK. e-mail: frederic.geissmann@kcl.ac.uk

Siamon Gordon is at Sir William Dunn School of Pathology, South Parks Road, Oxford OX1 3RE, UK. e-mail:siamon.gordon@path.ox.ac.uk

David A. Hume is at the Roslin Institute and Royal (Dick) School of Veterinary Studies, University of

Edinburgh, Roslin, Midlothian EH25 9PS, UK. e-mail:david.hume@roslin.ed.ac.uk

Allan M. Mowat is at the Glasgow Biomedical Research Centre, University of Glasgow, 120 University Place, Glasgow G12 8TA, UK. e-mail:a.m.mowat@clinmed.gla.ac.uk

Gwendalyn J. Randolph is at the Mount Sinai School of Medicine, New York, New York 10029, USA. e-mail: Gwendalyn.Randolph@mssm.edu doi: $10.1038 /$ nri2 2784 Published online 14 May 2010

Geissmann, F. et al. Development of monocytes, macrophages, and dendritic cells. Science 327 , 656-661 (2010).

2. Ajami, B., Bennett, J. L., Krieger, C., Tetzlaff, W. \& Rossi, F. M. Local self-renewal can sustain CNS microglia maintenance and function throughout adult life. Nature Neurosci. 10, 1538-1543 (2007).

3. Merad, M. \& Manz, M. G. Dendritic cell homeostasis. Blood 113, 3418-3427 (2009).

4. Chorro, L. et al. LC proliferation mediates neonatal development, homeostasis, and inflammationassociated expansion of the epidermal LC network J. Exp. Med. 206, 3089-3100 (2009)

5. Gordon, S. in Fundamental Immunology (ed Paul, W.) (Lippincott Williams and Wilkins, Philadelphia, 2008).

6. Pluddemann, A. \& Gordon, S. in Phagocyte-pathogen interactions (eds Russell, D. G. \& Gordon, S.) (American Society for Microbiology Press, Washington DC, 2009).

7. Gordon, S. \& Taylor, P. R. Monocyte and macrophage heterogeneity. Nature Rev. Immunol. 5, 953-964 (2005).

8. Mebius, R. E. \& Kraal, G. Structure and function of the spleen. Nature Rev. Immunol. 5, 606-616 (2005).

9. Swirski, F. K. et al. Identification of splenic reservoir monocytes and their deployment to inflammatory sites. Science 325, 612-616 (2009).

10. Steinman, R. M. Dendritic cells: versatile controllers of the immune system. Nature Med. 13, 1-5 (2007).

11. Naik, S. H. et al. Intrasplenic steady-state dendritic cell precursors that are distinct from monocytes. Nature Immunol. 7, 663-671 (2006).

12. Fogg, D. K. et al. A clonogenic bone marrow progenitor specific for macrophages and dendritic cells. Science 311, 83-87 (2006).

13. Liu, K. et al. Origin of dendritic cells in peripheral lymphoid organs of mice. Nature Immunol. 8 , 578-583 (2007).

14. Varol, C. et al. Monocytes give rise to mucosal, but not splenic, conventional dendritic cells. J. Exp. Med. 204 171-180 (2007).

15. Onai, N. et al. Identification of clonogenic common Flt3+M-CSFR + plasmacytoid and conventional dendritic cell progenitors in mouse bone marrow. Nature Immunol. 8, 1207-1216 (2007).

16. Jakubzick, C. et al. Lymph-migrating, tissue-derived dendritic cells are minor constituents within steadystate lymph nodes. J. Exp. Med. 205, 2839-2850 (2008)

17. Liu, K. et al. In vivo analysis of dendritic cell development and homeostasis. Science 324, 392-397 (2009).

8. Hume, D. A. The mononuclear phagocyte system. Curr. Opin. Immunol. 18, 49-53 (2006).

19. Hume, D. A. Differentiation and heterogeneity in the mononuclear phagocyte system. Mucosal Immunol. 1, 432-441 (2008)

20. Hume, D. A. Probability in transcriptional regulation and its implications for leukocyte differentiation and inducible gene expression. Blood 96, 2323-2328 (2000).

21. Hume, D. A. Macrophages as APC and the dendritic cell myth. J. Immunol. 181, 5829-5835 (2008).

22. Randolph, G. J., Inaba, K., Robbiani, D. F., Steinman, R. M. \& Muller, W. A. Differentiation of phagocytic monocytes into lymph node dendritic cells in vivo. Immunity 11, 753-761 (1999).
23 Serbina, N. V. Salazar-Mather, T. P., Biron, C. A. Kuziel, W. A. \& Pamer, E. G. TNF/iNOS-producing dendritic cells mediate innate immune defense against bacterial infection. Immunity 19, 59-70 (2003).

24. Steinman, R. M. \& Inaba, K. Stimulation of the primary mixed leukocyte reaction. Crit. Rev. Immunol. 5, 331-348 (1985)

25. Pavli, P., Woodhams, C. E., Doe, W. F. \& Hume, D. A Isolation and characterization of antigen-presenting dendritic cells from the mouse intestinal lamina propria. Immunology 70, 40-47 (1990).

26. Bogunovic, M. et al. Origin of the lamina propria dendritic cell network. Immunity 31, 513-525 (2009).

27. Denning, T. L., Wang, Y. C., Patel, S. R., Williams, I. R. \& Pulendran, B. Lamina propria macrophages and dendritic cells differentially induce regulatory and interleukin 17-producing T cell responses. Nature Immunol. 8, 1086-1094 (2007).

28. Pavli, P., Hume, D. A., Van De Pol, E. \& Doe, W. F. Dendritic cells, the major antigen-presenting cells of the human colonic lamina propria. Immunology 78 132-141 (1993).

29. Coombes, J. L. \& Powrie, F. Dendritic cells in intestinal immune regulation. Nature Rev. Immunol. 8, 435-446 (2008)

30. Platt, A. M. \& Mowat, A. M. Mucosal macrophages and the regulation of immune responses in the intestine. Immunol. Lett. 119, 22-31 (2008).

31. Laffont, S. \& Powrie, F. Immunology: dendritic-cell genealogy. Nature 462, 732-733 (2009).

32. Uematsu, S. et al. Regulation of humoral and cellular gut immunity by lamina propria dendritic cells expressing Toll-like receptor 5. Nature Immunol. 9, 769-776 (2008)

33. Mizoguchi, A. et al. Dependence of intestinal granuloma formation on unique myeloid DC-like cells. J. Clin. Invest. 117, 605-615 (2007).

34. Cerutti, A. \& Rescigno, M. The biology of intestinal immunoglobulin A responses. Immunity 28, 740-750 (2008).

35. Schulz, O. et al. Intestinal $\mathrm{CD} 103^{+}$, but not $\mathrm{CX}_{3} \mathrm{CR} 1^{+}$ antigen sampling cells migrate in lymph and serve classical dendritic cell functions. J. Exp. Med. 206, 3101-3114 (2009).

36. Niess, J. H. et al. CX $\mathrm{C}_{3} \mathrm{CR} 1$-mediated dendritic cell access to the intestinal lumen and bacterial clearance. Science 307, 254-258 (2005).

37. Rescigno, M., Lopatin, U. \& Chieppa, M. Interactions among dendritic cells, macrophages, and epithelial cells in the gut: implications for immune tolerance. Curr. Opin. Immunol. 20, 669-675 (2008).

38. Gonzalez-Juarrero, M., Shim, T. S., Kipnis, A. Junqueira-Kipnis, A. P. \& Orme, I. M. Dynamics of macrophage cell populations during murine pulmonary tuberculosis. J. Immunol. 171, 3128-3135 (2003).

39. Jakubzick, C. et al. Blood monocyte subsets differentially give rise to $\mathrm{CD} 103^{+}$and $\mathrm{CD} 103^{-}$ pulmonary dendritic cell populations. J. Immunol. 180, 3019-3027 (2008)

40. Idoyaga, J., Suda, N., Suda, K., Park, C. G. \& Steinman, R. M. Antibody to Langerin/CD207 localizes large numbers of $\mathrm{CD} 8 \alpha^{+}$dendritic cells to the marginal zone of mouse spleen. Proc. Natl Acad. Sci. USA 106 1524-1529 (2009).

41. Chieppa, M., Rescigno, M., Huang, A. Y. \& Germain, R. N. Dynamic imaging of dendritic cell extension into the small bowel lumen in response to epithelial cell TLR engagement. J. Exp. Med. 203 2841-2852 (2006).

42. Varol, C. et al. Intestinal lamina propria dendritic cell subsets have different origin and functions. Immunity 31, 502-512 (2009).

43. Niess, J. H. \& Adler, G. Enteric flora expands gut lamina propria $\mathrm{CX}_{3} \mathrm{CR} 1$ + dendritic cells supporting inflammatory immune responses under normal and inflammatory conditions. J. Immunol. 184 2026-2037 (2010)

44. Kissenpfennig, A. et al. Dynamics and function of Langerhans cells in vivo: dermal dendritic cells colonize lymph node areas distinct from slower migrating Langerhans cells. Immunity 22, 643-654 (2005).

45. Keshav, S., Chung, P., Milon, G. \& Gordon, S Lysozyme is an inducible marker of macrophage activation in murine tissues as demonstrated by in situ hybridization J. Exp. Med. 174, 1049-1058 (1991).

46. Berlin, I. The hedgehog and the fox: an essay on Tolstoy's view of history (Weidenfeld and Nicolson, London, 1953).

47. Steinman, R. M., Hawiger, D. \& Nussenzweig, M. C. Tolerogenic dendritic cells. Annu. Rev. Immunol. 21, 685-711 (2003) 
48. Iliev, I. D., Mileti, E., Matteoli, G., Chieppa, M. \& Rescigno, M. Intestinal epithelial cells promote colitisprotective regulatory T-cell differentiation through dendritic cell conditioning. Mucosal Immunol. 2, 340-350 (2009)

49. Mellman, I. \& Steinman, R. M. Dendritic cells: specialized and regulated antigen processing machines. Cell 106, 255-258 (2001).

50. Martinez, F. O., Sica, A., Mantovani, A. \& Locati, M. Macrophage activation and polarization. Front. Biosci. 13, 453-461 (2008)

51. Inaba, K. et al. Granulocytes, macrophages, and dendritic cells arise from a common major histocompatibility complex class II-negative progenitor in mouse bone marrow. Proc. Natl Acad. Sci. USA 90, 3038-3042 (1993).

52. Auffray, C., Sieweke, M. H. \& Geissmann, F. Blood monocytes: development, heterogeneity, and relationship with dendritic cells. Annu. Rev. Immuno 27, 669-692 (2009).

53. Fleetwood, A. J., Lawrence, T., Hamilton, J. A. \& Cook, A. D. Granulocyte-macrophage colonystimulating factor (CSF) and macrophage CSFdependent macrophage phenotypes display differences in cytokine profiles and transcription factor activities: implications for CSF blockade in inflammation. J. Immunol. 178, 5245-5252 (2007).

54. MacDonald, K. P. et al. The colony-stimulating factor receptor is expressed on dendritic cells during differentiation and regulates their expansion. J. Immunol. 175, 1399-1405 (2005).

55. Hume, D. A. Immunohistochemical analysis of murine mononuclear phagocytes that express class II major histocompatibility antigens. Immunobiology 170 , 381-389 (1985)

56. Steinman, R. M. \& Banchereau, J. Taking dendritic cells into medicine. Nature 449, 419-426 (2007).

57. Nahrendorf, M. et al. The healing myocardium sequentially mobilizes two monocyte subsets with divergent and complementary functions. J. Exp. Med. 204, 3037-3047 (2007).

58. Movahedi, K., et al. Identification of discrete tumorinduced myeloid-derived suppressor cell subpopulations with distinct T cell-suppressive activity. Blood 111, 4233-4244 (2008).

59. Henri, S. et al. CD207+ CD103+ dermal dendritic cells cross-present keratinocyte-derived antigens irrespective of the presence of Langerhans cells. J. Exp. Med. 207 , 189-206 (2010).

60. Bonifaz. et al. In vivo targeting of antigen to maturing dendritic cells via the DEC-205 receptor improves T cell vaccination. J. Exp. Med. 199, 815-824 (2004).

61. Longhi, M. P. et al. Dendritic cells require a systemic type I interferon response to mature and induce CD4 ${ }^{+}$Th 1 immunity with poly IC as adjuvant. J. Exp. Med. 206, 1589-1602 (2009).

62. Patsouris, D. et al. Ablation of CD11c-positive cells normalizes insulin sensitivity in obese insulin resistant animals. Cell Metab. 8, 301-309 (2008)

63. Haniffa, M. et al. Differential rates of replacement of human dermal dendritic cells and macrophages during hematopoietic stem cell transplantation. J. Exp. Med. 206, 371-385 (2009).

64. Liu, K. et al. In vivo analysis of dendritic cell development and homeostasis. Science 324 392-397 (2009).

65. Naik, S. H. et al. Development of plasmacytoid and conventional dendritic cell subtypes from single precursor cells derived in vitro and in vivo. Nature Immunol. 8, 1217-1226 (2007).

Competing interests statement

The authors declare no competing financial interests.

DATABASES

UniProtKB: http://www.uniprot.org

CD8a $\mid$ CD11b $\mid$ CD11c $\mid$ CD103|CSF1 $\mid$ CSF1R $\mid C X_{3}$ CR1

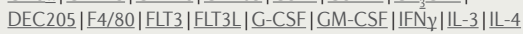

\section{FURTHER INFORMATION}

Frédéric Geissmann's homepage: http://kcl.ac.uk/schools/ Siamon Gordon's homepage: http://users.path.ox.ac.

uk/ cholt/

David A. Hume's homepage: http://www.roslin.ed.ac.uk/ Allan M. Mowat's homepage : http://www.gla.ac.uk/ departments/immunology/researchgroups/allanmowat/ Gwendalyn J. Randolph's homepage:

http://www.mountsinai.org/profiles/gwendalyn-jrandolph?id $=0000072500001497156102$

ALL LINKS ARE ACTIVE IN THE ONLINE PDF 